\title{
Retinopathy of prematurity in practice. I: screening for threshold disease
}

R Brennan, L Gnanaraj and DG Cottrell

\begin{abstract}
Aims To review an 11-year period of screening for retinopathy of prematurity (ROP) in the north of England by a single ophthalmologist. To identify the gestational ages and birth weights of babies reaching different stages of ROP. To investigate the workload involved in screening to detect threshold ROP, and that the practical outcomes had narrower inclusion criteria for screening. To identify babies treated for threshold disease.

Methods During the period August 1987October 1998, babies were screened according to the national guidelines and the results were prospectively entered onto a computerised database. These data were then systematically reviewed.
\end{abstract}

Results Data were available for 484 babies: 203 (41.9\%) developed any ROP, 46 (9.5\%) reached stage 3 ROP, and $25(5.2 \%)$ reached threshold and were treated. Data on 425 babies showed them to require an average of 2.3 screening examinations per baby. It took an average of 39 screening examinations to detect one case of threshold ROP. The more premature and lighter birth weight babies required the most examinations. Therefore, restricting the inclusion criteria for screening would only have reduced the total number of screenings modestly and could have allowed us to miss two of our threshold cases who were both of 30 weeks gestational age and $>1400 \mathrm{~g}$ birth weight.

Conclusions Screening is time consuming but worthwhile in view of the benefits of treatment. As applied to babies in the north of England, the current national screening criteria are satisfactory. The results of treatment of the babies identified in this study are presented in the accompanying paper. Eye (2003) 17, 183-188. doi:10.1038/ sj.eye. 6700296
Keywords: retinopathy of prematurity (ROP); gestational age; birth weight

\section{Introduction}

Screening for 'threshold' retinopathy of prematurity (ROP) has become obligatory following the confirmation of a significant benefit from treatment. ${ }^{1}$ The criteria for which babies to screen, and when, remain controversial.

As previous studies have shown a geographical variation in the incidence of threshold ROP in babies born at similar gestational ages, ${ }^{2-4}$ it is important to continue to analyse screening data from different regions in order to inform the debate. It is likely that screening criteria will have to be specifically defined for particular populations. Furthermore, as neonatal practices evolve, the criteria will require periodic review.

A previous study showed an incidence of $3.6 \%$ of threshold ROP in babies born at less than 32 weeks in the Northern Region of England. ${ }^{5}$ However, that study was not able to provide detailed data on the specific risk at different gestational ages, and neither did it provide any measure of the workload required to screen such babies. This is relevant in the context of the current debate about the equipment and personnel required to perform ROP screening.

The present study was planned to provide these data for the screening practice of a single ophthalmologist (DGC) in a provincial teaching hospital over an 11-year period. It also allowed the identification of all babies treated by the same ophthalmologist over this period, thereby providing the basis for the accompanying paper describing the long-term results of treatment. ${ }^{6}$
Department of Ophthalmology Royal Victoria Infirmary Newcastle upon Tyne UK

Correspondence: DG Cottrell Department of Ophthalmology Royal Victoria Infirmary Queen Victoria Road Newcastle upon Tyne NE1 4LP, UK

Tel: +44 1912825450 Fax: +44 1912275246 E-mail: d.g.cottrell@ ncl.ac.uk

Received: 30 July 2001 Accepted: 7 May 2002

Presented in part at the 'International Symposium on Retinopathy of Prematurity', Taormina Italy, 21-24 September 1997 


\section{Methods}

Data on babies screened between August 1987 and October 1998 were recorded in accordance with the International Classification of $\mathrm{ROP}^{7}$ and entered prospectively on a computerised database. Efforts were made to screen appropriate infants according to the criteria in use at the time, which were:

1987-1990: birth weight (BW) $\leq 1500$ g;

1990-1998: BW $\leq 1500 \mathrm{~g}$ and/or gestational age (GA) at birth $\leq 31$ weeks. $^{8}$

Babies were examined at 6 weeks chronological age (CA) or 36 weeks postmenstrual age (PMA), whichever was earlier, and re-examined at least biweekly until the retina was vascularised into zone III with no ROP above stage 2 . When the signs indicated that ROP was approaching threshold, examinations were conducted up to twice weekly. Threshold ROP was defined as at least five contiguous or eight cumulative clock hours of stage 3 ROP in zone I or II in the presence of plus disease. ${ }^{9}$ The diagnosis of threshold disease resulted in treatment being offered, as reported in the accompanying paper. ${ }^{6}$

Screening was performed by indirect ophthalmoscopy using a 28 D lens, with a lid speculum and scleral indentor under topical anaesthesia. Pupil dilatation was achieved by instilling $0.5 \%$ tropicamide and $2.5 \%$ phenylephrine to both eyes, 60 and $30 \mathrm{~min}$ prior to the examination.

The main special care units involved were those in the Newcastle upon Tyne hospitals (where allocation of cases was shared on a random basis with one colleague) and in Gateshead. Certain units in neighbouring hospitals also requested screening from time to time. Once allocated, individual cases generally remained under the care of that ophthalmologist, so that the present study represents a personal series (by DGC). The only exceptions were during periods of leave (totalling 8 weeks/year) when colleagues were deputised to maintain the service, and in cases when babies were transferred to or from other units in the region when local staff began or completed the screening as appropriate. In these cases, prior or follow-up data were sought from these colleagues.

The data were analysed to determine the following:

(1) the maximum stage of ROP recorded in each eye during the screening process;

(2) the degree of symmetry between the two eyes of each baby;

(3) the GA and BW of babies reaching each maximum stage of ROP;

(4) the CA and PMA at which the maximum stage was recorded; and
(5) the number of screening examinations required for each baby, either to diagnose threshold disease or to determine that the baby was safe for discharge from the screening programme.

\section{Results}

According to the above criteria, 503 babies were screened; of these, 19 were excluded from the analysis because of incomplete data. In addition to these 484 babies, 10 were referred in from other screening programmes for treatment, having reached threshold disease. Excluding those babies referred in, 41.9\% $(n=203)$ developed acute ROP. A total of $19.6 \%(n=95)$ reached a maximum of stage $1,12.8 \%(n=62)$ reached stage $2,9.5 \%$ $(n=46)$ reached stage 3 , and $5.2 \%(n=25)$ reached threshold for treatment. Of the babies referred in, one had reached stage 4 in one eye and stage 3 in the other.

Charts 1 and 2 show the maximum stage of ROP in the worst affected eye of each baby, analysed by GA and BW respectively. In most cases, the severity of ROP was symmetrical, but in $11.3 \%(n=56)$ there was one stage difference between the two eyes and in $1.6 \%(n=8)$ two stage differences. In $60.9 \%$ of the asymmetric cases, the right eye was the worst affected. Of those that had two stage differences, six had no ROP in one eye and stage 2 in the other, and two had stage 3 in one eye and stage 1 in the other. Of the 35 babies who required treatment, two had unilateral threshold disease with subthreshold stage 3 in the other eye.

Chart 3 and 4 show the CA and PMA, respectively, at which threshold was reached. The age range at which threshold was reached was 6-17 weeks CA and 34-40

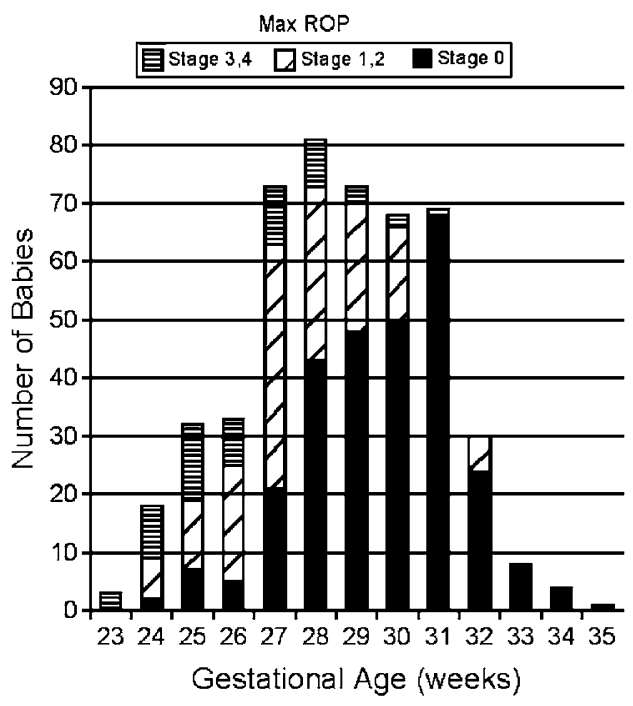

Chart 1 GA distribution and maximum stage of ROP reached in 494 babies. The stage in the worst affected eye is represented. 


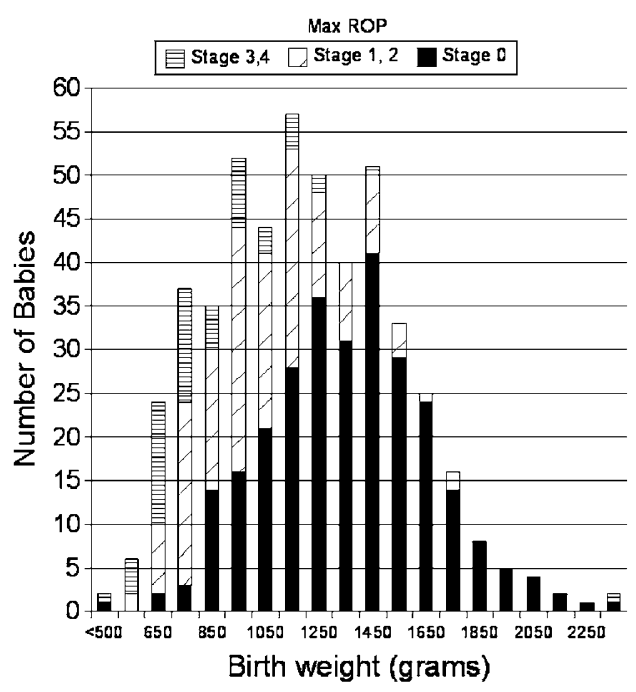

Chart 2 BW distribution and maximum stage of ROP reached in 494 babies. The stage in the worst affected eye is represented. Mid-range value for each alternate BW group is shown.

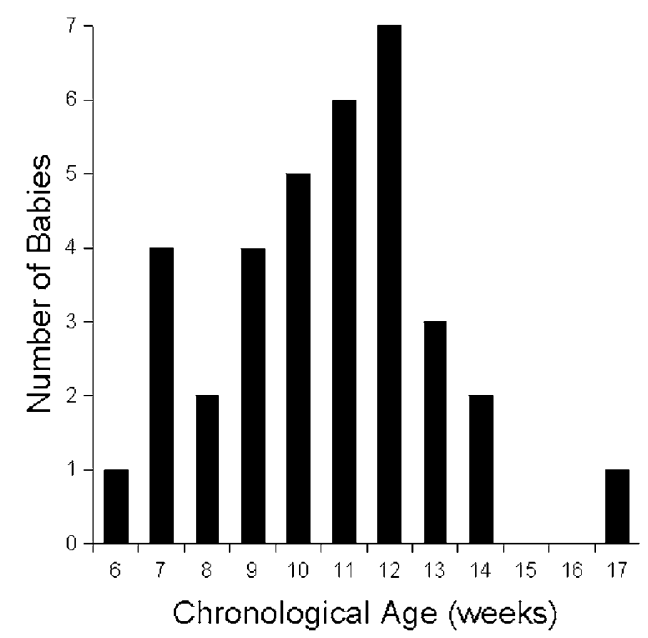

Chart 3 CA at which threshold was reached in 35 babies. In one case, the right eye reached threshold at 12 weeks and the left at 14 weeks. The value for the right eye is graphed.

weeks PMA. In the 33 babies who had bilateral treatment, threshold was detected at the same time in both eyes except in one case, who reached threshold in the right eye at 12 weeks CA and 37 weeks PMA and in the left eye 2 weeks later. The timings for the right eye are used in the charts.

In the analysis of screening workload, all babies in part screened by others have been excluded to ensure absolute accuracy of the data. This leaves 425 babies. They were screened in total 969 times. Out of this group 25 babies reached threshold. It, therefore, took an average of 39 screening examinations to detect one case of

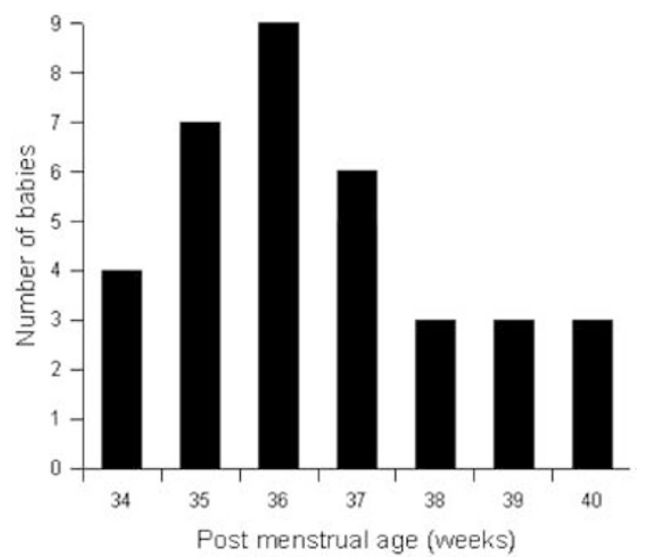

Chart 4 PMA at which threshold was reached in 35 babies. In one case, the right eye reached threshold at 37 weeks and the left at 39 weeks. The value for the right is graphed.

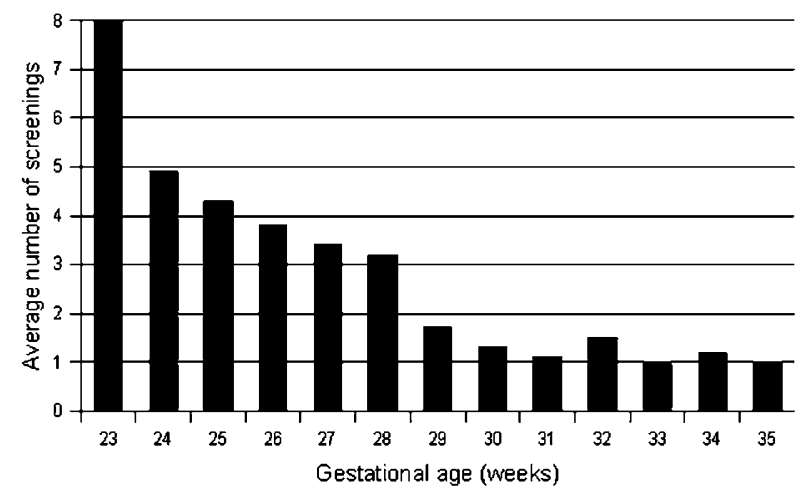

Chart 5 Average number of screenings performed per GA group in 425 babies.

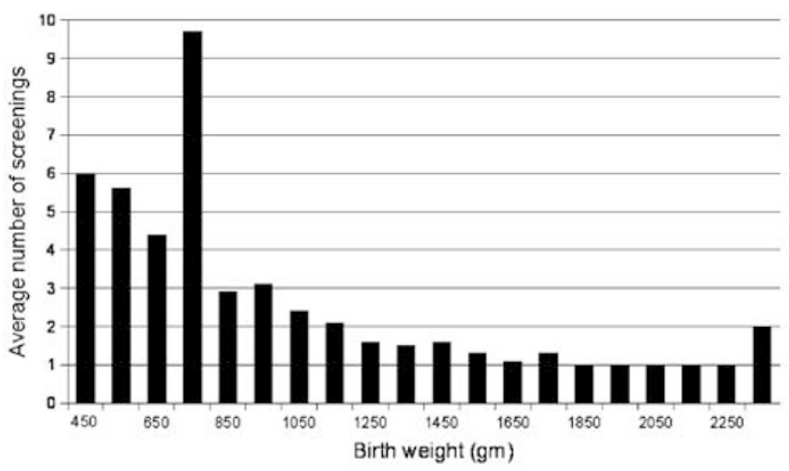

Chart 6 Average number of screenings performed per BW group in 425 babies. Mid-range value of each alternate BW group is shown.

threshold ROP. The 25 babies who reached threshold were screened in total 104 times.

Charts 5 and 6 show the workload required to screen 425 babies in terms of the average number of 
examinations required as a function of GA and BW respectively.

\section{Discussion}

\section{Incidence of ROP}

The reported incidence of ROP varies across the UK between regions and even between units, suggesting that caution must be applied in extrapolating conclusions about screening criteria from one population to another. In the present study, acute ROP of any stage was found in $41.9 \%$ and stage 3 disease in $9.5 \%$ of screened babies. This contrasts with acute ROP in 50.9\% and stage $3 / 4$ ROP in $4.7 \%$ reported from Leicester, Nottingham, and Derby between 1985 and $1987^{10}$ and stage 3 ROP in $2.2 \%$ of Birmingham babies studied between 1989 and $1995 .{ }^{4}$ It is to be expected that observer bias may influence the results of studies of this nature, but these two particular reports involved the same ophthalmologist as screener. Patient demographics may have an effect, as ROP increases in severity with reducing BW and GA. However, patient demographics were little different in these two studies and in the present study. Bias in patient inclusion criteria may also be important. Some units will classify very immature babies as live-births and treat with intensive care while others treat most of these babies as stillbirths. This may have resulted in a significantly higher rate of severe ROP, but a significantly lower actual death rate per 1000 births in one English city compared with four other English cities. ${ }^{11}$ The quality of neonatal care is also sometimes regarded as affecting the incidence of ROP and may well be relevant in the increased incidence of severe disease in larger infants in Eastern Europe. ${ }^{12}$ However, quite marked differences between two hospitals in a Copenhagen-based study proved hard to explain: acute ROP was observed in $26 \%$ and threshold in $3.4 \%$ in one hospital compared with acute ROP in $46 \%$ and threshold in $10.7 \%$ in the other hospital. No apparent reason was found and it was postulated that the difference was an artefact of the small numbers involved (147 and 56 babies, respectively). ${ }^{2}$

An audit of screening throughout the Northern Region of England ${ }^{13}$ has indicated that compliance with screening guidelines during a major part of our study period was poor. The study can therefore not be regarded as a cohort study. It is encouraging, however, that all babies screened during a 2-year window of the present study (born during 1990-1991) were re-examined at age 2 as part of another project; ${ }^{5}$ no baby pronounced free of significant ROP at screening in our study was found to have developed cicatricial disease subsequently (PM Pennefather, personal communication). This provides a limited validation of the accuracy of our screening.

\section{Age at onset of maximum ROP}

Quinn et $_{\text {al }}{ }^{14}$ correlated postnatal and postconceptual age of onset of ROP and found that infants born earlier in gestation develop the first signs of ROP at a later postnatal age but earlier postconceptual age than infants born later in gestation. Some have found that there is a narrower range of $\mathrm{CA}^{15}$ and others a narrower range of $\mathrm{PMA}^{3,16}$ for the onset of the maximum stage of ROP. In our study, PMA gave a narrower range than CA (6 weeks cf 11 weeks).

Opinion varies as to when screening of babies should begin; this is reflected in conflicting recommendations in different guidelines. The range is from as early as $4-6$ weeks CA (or 31-33 weeks postconceptual age) in one paper $^{17}$ to as late as 8-10 weeks CA in another. ${ }^{18}$ In our study, one baby had reached threshold by his first exam at $6 \frac{1}{2}$ weeks (CA) and another study reported stage 3 developing as early as 5 weeks postnatal age. ${ }^{15}$ As the earliest case of threshold disease was detected at $6 \frac{1}{2}$ weeks CA, the policy of commencing screening at 6 weeks CA would seem safe for our population. We feel that using CA rather than PMA to determine when screening begins is inherently more satisfactory as it is securely defined; PMA, of course, relies on the accuracy of the GA assessment.

\section{Definition of threshold ROP}

We used the same definition as the Cryotherapy for Retinopathy of Prematurity (CRYO-ROP) Study for Threshold ROP: at least five contiguous or eight cumulative clock hours of stage 3 ROP in zone I or II with plus disease. ${ }^{9}$ There are other definitions of threshold disease in use, for example, the broader UK guidelines which in addition to the above include five contiguous or eight cumulative clock hours of stage 3 ROP in zone III with plus disease. ${ }^{19}$ The STOP-ROP study had different criteria for zones I and II threshold ROP; for zone II disease it used the same definition as the CRYO-ROP study, but in zone I it defined as 'threshold' any stage of ROP with plus disease, or stage 3 ROP with or without plus disease. ${ }^{20}$

\section{ROP asymmetry}

In our study, $12.9 \%(64 / 494)$ of babies had asymmetry of acute phase ROP with $1.6 \%(n=8)$ being asymmetrical by two stages. Of those that reached threshold ROP, 5.7\% $(2 / 35)$ had unilateral threshold ROP. In the CRYO-ROP 
study, $17.5 \%$ (51/291) had unilateral threshold ROP, ${ }^{21}$ Fielder et $a l^{10}$ found that $25.4 \%(74 / 291)$ cases of acute ROP were asymmetrical but by only one stage.

\section{Screening inclusion criteria}

There is a potential for reducing workload by reducing the upper inclusion limits of BW and GA. In particular, Goble et al $^{4}$ retrospectively reviewed 1429 babies screened for ROP who fulfilled the Royal College of Ophthalmologists current guidelines. They found if stricter inclusion criteria for screening had been used (babies with a BW $\leq 1250 \mathrm{~g}$ and/or GA $\leq 29$ weeks), no babies with stage 3 ROP would have been missed and $30 \%$ fewer babies could have been examined. If in the present study these criteria had been used, $29 \%$ fewer babies would have been screened. This would, however, have reduced the number of screening examinations by only $15 \%$ as the screening workload decreases with increasing GA and BW (see Charts 5 and 6). Of concern in our series is that two babies would have been missed using these criteria; one baby (referred in) was $1440 \mathrm{~g}$ at birth and 30 weeks $\mathrm{GA}^{22}$ and the other $2300 \mathrm{~g}$ and 30 weeks GA. The latter's mother was diabetic. Neither baby fitted the additional sickness criteria suggested by Goble et al.

\section{Nonophthalmologists and ROP screening}

Alternatives to an ophthalmologist screening all at-risk babies have been investigated. Saunders et $a l^{23}$ compared the reliability of a paediatrician's assessment of vessel dilatation and tortuosity at the posterior pole with that of an ophthalmologist. They then looked at the

ophthalmologist's peripheral retinal findings and found no babies with prethreshold disease or worse would have been missed if the ophthalmologist were only to have examined those with abnormal posterior poles or inadequately visualised posterior poles. This would have cut down the number of babies needing to be seen by an ophthalmologist by $33 \%(47 / 142) .{ }^{23}$ However, it is being suggested that babies may have a better visual outcome if treated earlier than the conventional threshold point. This is being investigated by the Early Treatment for Retinopathy of Prematurity Study. One of the entry criteria is zone II ROP with stage 3 but not plus disease. ${ }^{24}$ If this should prove beneficial, direct ophthalmoscopy would not be sufficient to diagnose critical disease. Also, the paediatrician's evaluation involved dilated funduscopy and the use of a paediatric speculum, so physiological stress to the baby may not have been much reduced. ${ }^{25}$

There is a training issue here. If nonophthalmologists took over most ROP screening, the experience of ophthalmologists in the field would be reduced and training of new ophthalmologists would be difficult. Furthermore, although it is difficult to quantify, it is the experience of the authors that subtle changes in retinal findings on serial examinations provide valuable additional data to assist the assessment of disease at the subthreshold/threshold border.

\section{ROP screening workload}

In this study, the diagnosis of 25 cases of threshold disease required 969 examinations. In our experience, the average time required for one examination is $30 \mathrm{~min}$ (to include times for travelling and paperwork). We, therefore, estimate that $19 \mathrm{~h}$ of ophthalmologists' time are required to detect one threshold case. According to the CRYO-ROP trial, the risk of an adverse functional outcome in threshold disease without treatment is $61.7 \% .^{26}$ The accompanying paper on treatment of $\mathrm{ROP}^{6}$ indicates that with modern laser therapy, an adverse functional outcome occurs in only $10 \%$ of eyes. The costeffectiveness of screening and cryotherapy for threshold disease has been elegantly calculated by Javitt et al. ${ }^{27}$ Our data, in conjunction with Javitt et al's calculations, show that the economic cost of the ophthalmologists time is amply justified in terms of saving an individual from a lifetime of blindness.

\section{Summary}

The experience of one ophthalmologist in screening for ROP over a period of approximately 11 years has been presented. Useful conclusions can be drawn to inform the current debate on the appropriate inclusion criteria for screening and the cost-effectiveness of the process. The data support the suggestion that the current criteria for the UK are effective but not excessive.

\section{Acknowledgements}

No research funding was used for this study. The authors have no proprietary interest in any of the materials used in this study.

\section{References}

1 Multicentre Trial of Cryotherapy for Retinopathy of Prematurity. One-year outcome-structure and function. Arch Ophthalmol 1990; 108: 1408-1416.

2 Fledelius HC, Kjer B, Rosenberg T. Retinopathy of prematurity in a Copenhagen high-risk sample. 1997-98. Acta Ophthalmol Scand 2000; 78: 362-365. 
3 Holmström G, El Azazi M, Jacobson L, Lennerstrand G. A population based, prospective study of the development of ROP in prematurely born children in the Stockholm area of Sweden. Br J Ophthalmol 1993; 77: 417-423.

4 Goble RR, Jones HS, Fielder AR. Are we screening too many babies for retinopathy of prematurity? Eye 1997; 11: 509-514.

5 Pennefather PM, Clarke MP, Strong NP, Cottrell DG, Fritz S, Tin W. Ocular outcome in children born before 32 weeks gestation. Eye 1995; 9(Suppl): 26-36.

6 Gnanaraj L, Brennan R, Cottrell DG. Retinopathy of prematurity in practice. II: long term results following treatment for threshold disease. Eye 2003; 17: 187-191.

7 Committee for the Classification of Retinopathy of Prematurity. An international classification of retinopathy of prematurity. Br J Ophthalmol 1984; 68: 690-697.

8 Anon. College news: ROP screening duty. Q Bull Coll Ophthalmol 1990; Autumn: 6.

9 Cryotherapy for Retinopathy of Prematurity Cooperative Group. Multicenter Trial of Cryotherapy for Retinopathy of Prematurity; preliminary results. Arch Ophthalmol 1988; 106: 471-479.

10 Fielder AR, Shaw DE, Robinson J, Ng YK. Natural history of retinopathy of prematurity: a prospective study. Eye 1992; 6: 233-242.

11 Vyas J, Field D, Draper ES et al. Severe retinopathy of prematurity and its association with different rates of survival in infants of less than $1251 \mathrm{~g}$ birth weight. Arch Dis Child Fetal Neonatal Ed 2000; 82: F145-F149.

12 Bagdoniene R, Surtautiene R. Threshold retinopathy of prematurity in Lithuania. In: Reibaldi A, Di Pietro M, Scuderi A, Malerba E (eds). Progress in Retinopathy of Prematurity. Kugler Publications: Amsterdam/New York, 1997, pp 31-36.

13 Ziakas NG, Cottrell DG, Milligan DWA, Pennefather PM, Bamashmus MA, Clarke MP. Regionalisation of retinopathy of prematurity (ROP) screening improves compliance with guidelines: an audit of ROP screening in the Northern Region of England. Br J Ophthalmol 2001; 85: 807-810.

14 Quinn GE, Johnson L, Abbasi S. Onset of retinopathy of prematurity as related to postnatal and postconceptional age. Br J Ophthalmol 1992; 76: 284-288.

15 Schalij-Delfos NE, Zijlmans BLM, Wittebol-Post D, Tan KEWP, Cats BP. Screening for retinopathy of prematurity: do former guidelines still apply? J Pediatr Ophthalmol Strabismus 1996; 33: 35-38.
16 Fledelius HC, Dahl H. Retinopathy of prematurity, a decrease in frequency and severity. Trends over 16 years in a Danish county. Acta Ophthalmol Scand 2000; 78: 359-361.

17 A joint statement of the American Academy of Pediatrics, the American Association for Pediatric Ophthalmology and Strabismus, and the American Academy of Ophthalmology. Screening examination of premature infants for retinopathy of prematurity. Ophthalmology 1997; 104: 888-889.

18 Flynn JT. Optimal timing of examination for acute retrolental fibroplasia. Ophthalmology 1981; 88: 667-668.

19 Wilkinson AR, Clark D, Fielder A et al. Retinopathy of prematurity: guidelines for screening and treatment. The report of a joint working party of the Royal College of Ophthalmologists and the British Association of Perinatal Medicine. Early Hum Dev 1996; 46: 239-258.

20 Supplemental Therapeutic Oxygen for Prethreshold Retinopathy of Prematurity (STOP-ROP), a randomized, controlled trial. I: primary outcomes. Pediatrics 2000; 105(2): 295-310.

21 Cryotherapy for Retinopathy of Prematurity Cooperative Group. Multicenter Trial of Cryotherapy for Retinopathy of Prematurity. Ophthalmological outcomes at 10 years. Arch Ophthalmol 2001; 119: 1110-1118.

22 Pollock W, Inglesby DV, Cottrell DG. Retinopathy of prematurity: guidelines for screening and treatment (letter; comment). Eye 1998; 12: 1035.

23 Sauders RA, Donahue ML, Berland JE, Roberts EL, Von Powers B, Rust PF. Non-ophthalmologist screening for retinopathy of prematurity. Br J Ophthalmol 2000; 84: 130-134.

24 Good WV, Hardy RJ. The Multicenter Study of Early Treatment for Retinopathy of Prematurity (ETROP). Ophthalmology 2001; 108(6): 1013-1014.

25 Laws DE, Morton C, Weindling, Clark D. Systemic effects of screening for retinopathy of prematurity. $\mathrm{Br} J$ Ophthalmol 1996; 80: 425-428.

26 Cryotherapy for Retinopathy of Prematurity Cooperative Group. Multicenter Trial of Cryotherapy for Retinopathy of Prematurity: Snellen visual acuity and structural outcome at $5 \frac{1}{2}$ years after randomisation. Arch Ophthalmol 1996; 114: 417-424.

27 Javitt J, Dei Cas R, Chiang YP. Cost-effectiveness of screening and cryotherapy for threshold retinopathy of prematurity. Pediatrics 1993; 91(5): 859-866. 